\title{
CCD IMAGERS FOR ASTRONOMY: PAST PROBLEMS AND FUTURE HOPES
}

\author{
John C. Geary \\ Smithsonian Astrophysical Observatory \\ 60 Garden St. \\ C'ambridge, MA 02138
}

\section{Introduction: The long trek.}

The search for high-quality, large-format CCD imagers during the 1980's reminds me of a journey across a large and forbidding desert. One starts off full of great expectations of a quick completion, but discouragement sets in around mid-journey as the barrenness of the landscape becomes apparent and the goal on the horizon is shown to be only a mirage. Finally, mountains resolve themselves from the mirages and we take heart in knowing that we seem finally to be making some progress. I think the analogy fits the CCD situation well, starting with the view from the time period $1979-80$ and working toward the present.

Back at the beginning, spirits were high and everyone looked forward to the new vistas offered by the emerging technology, with every expectation that progress in CCD technology would quickly yield up near-perfect devices for our demanding astronomical applications. We had, for instance, the RCA 320 X 512 backside-illuminated chip, which, despite its mediocre noise and charge-transfer performance, remains virtually unexcelled in quantum efficiency to this day. GEC in Britain was in the process of developing a promising low-noise device, and of course, there were tremendous expectations for the Space Telescope $800 \mathrm{X}$ 800 imagers from TI, although the secrecy surrounding their performance and availability was a most annoying feature for those of us not in the ST loop. With such a good start in both the commercial TV sphere (RCA, GEC, and later Thomson) and the non-commercial specialty product development (TI), we had every reason to suppose that our ideal imagers were close at hand. Then the landscape began drying up.

What happened to those of us involved in CCD instrument development during the period of 1981 - 87 can only be described as a descent into ever-increasing depression at the lack of progress in device availability, punctuated be brief moments of hope from time to time. Things started to go bad when we discovered how hard it was to interact with the major commercial producers to try to combine into one imager all the desireable features that had been demonstrated individually. What we wanted was the high quantum efficiency of the RCA backside- illuminated CCD combined with the low noise and good charge transfer of devices like those of GEC (later produced by EEV) or Thomson. What we got was indifference on the part of the manufacturers, who were locked in a mostly losing battle with the Japanese to develop the solid-state hand-held color TV camera. RCA made one largely unsuccessful attempt to produce a scientific grade device based on their commercial 
chip, but along the way a decision was made to terminate CCD development entirely, and they have sadly passed from the scene. EEV for years resisted pleas to develop a thinning process for their devices so that high blue quant um efficiency might be realized in a backsideilluminated mode. Their one excursion into large-area devices (1500 X 1500 pixels) for scientific applications has evidently not been very successful, as I have heard nothing of it for several years. Thomson has also been reluctant to try thinning until recently, but has remained at least a stable supplier of one useful imager over the past few years. Finally, to the great disappointment of all concerned, TI chose early on to terminate any further work on the technology developed for the Space Telescope imagers, leaving the astronomical community with only a few crumbs from what was hoped would be a great banquet.

We have occasionally had our spirits lifted by the prospect of real progress in putting the CC'D puzzle together right. Thanks to the efforts of Jim Janesick and co-workers at JPL, the two great problems plaguing the TI imagers, namely deferred charge and unstable quantum efficiency (quantum efficiency hysteresis), have been largely solved, the first by a cleaver change in the clocking scheme and the latter by various surface treatments such as UV floods or thin platinum overcoats. At the same time, concerted efforts by a consortium of research institutions and the National Science Foundation resulted in at least a few of the devices rejected from the space program being made available for astronomical research. Alas, many of these imagers proved to be of the "overly thick" variety, having low blue quantum efficiency and not responding well to surface treatments. They have, however, whetted our appetites enormously, as their other characteristics (noise and charge transfer) are often superb.

The other event that has from time to time lead to great expectations has been the formation of a team at Tektronix to produce scientific CCD imagers with both outstanding performance and large (even huge) formats. Beginning in $1984-85$, it was learned that this effort would indeed be aimed at producing low-noise thinned devices, initially in $512 \times 512$ format with quite substantial pixels ( 27 microns) and subsequently in a truly awesome 2048 $\mathrm{X} 2048$ size. By mid-1986, the first experimental chips became available for initial testing, and it was then that a new problem, large numbers of traps called "pockets", became evident, rendering most devices useless for precision work. Once again our hopes for a speedy end to the C'CD drought were dashed, as Tektronix was forced back into funclamental investigations of the chemistry and physics involved instead of device production.

\subsection{The Recent Past: Hope on the horizon?}

Beginning 18 months ago or so and continuing right up to the present, several developments have occurred that appear to have some substance to them. Compared to the preceding 6 or 7 years, one can certainly say that there is at last some progress to cheer. These developments arise from several independent efforts and involve a variety of players, giving us some reason to believe that we will at last have access to scientific grade imagers of moderate to large format, even if one or more of these projects ultimately fail.

The first new development to catch our attention was the use of a so-called "silicon foundry" by Photometrics Ltd. to produce for them under contract a 516 X 516 CCDD, which has subsequently been shown to possess outstanding charge transfer and noise performance. By concentrating solely on the technical aspects of device production, not subsequent marketing considerations, the silicon foundry can provide its contracting customer with a product tailored to specific needs. Entry level costs for such best-effort projects run several hundred thousand dollars, too high for most institutions alone, but increasingly 
within reach of a consortia of institutions operating in a cooperative fashion. At least two silicon foundries advertise special competence in CCD technology and more may eventually be added to the list.

At about the same time, Photometrics announced the commercial availability of a phosphor coating which could be applied by vacuum sublimation. This offers the possibility of getting significant response in the blue / UV spectral regions from devices that otherwise are weak there, such as unthinned frontside-illuminated imagers and perhaps also the "overly-thick" TI devices.

The National Science Foundation has funded a grant through Lick Observatory to develop a large CCD imager at Reticon Corp. The format chosen is $400 \times 1200$, with large 27 micron pixels. While ideal for many spectroscopic applications, this device should also prove interesting for direct imaging programs as well. Some of the initial experimental devices have already been tested and shown to be very promising.

Also, a new push at Thomson CSF toward production of scientific grade CCD's is in evidence. A few preliminary data sheets on their 512 X 512 and 1024 X 1024 developmental devices have been circulated and there is now talk of progress in thinning, to be ultimately marle available on these imagers.

Finally, the long ordeal at Tektronix may be coming to an end, as they are now shipping 512 X 512 CCD's to back-ordered customers. It seems that the infamous pocket problem may finally be solved, or at least reduced to a manageable level, and testing is now under way at several locations to verify this. If so, then we can expect to start seeing the first. high-grade $2048 \times 2048$ devices in the not so distant future, perhaps even in a matter of a few months.

\subsection{The Immediate Future and Beyond:}

Those faced with making decisions on specific CCD's to buy in the next year or two will at least have the Iuxury of a nonzero number of options available. I have listed below some of the best bets for the near future, starting with those that are essentially "off the shelf" and working on to those still in development, which include all of the really large devices. The actual choice of one device over another will depend mostly on system requirements and availability of the requisite cosmetic quality, not so much on the cost of the particular CCD itself, except in the case of truly huge formats such as the large Tektronix device. This reflects the often unappreciated fact that, for the most part, the cost of the CCD itself (when finally available ) is usually a small fraction of the total cost of the camera system. This is especially true when one takes into account the hardware and software burden necessary to support electronic imaging and subsequent reductions.

\section{Photometrics 516 X 516:}

20 micron pixels; $10.2 \mathrm{~mm}$ square format.

Unthinned, frontside-illumination; can be phosphor coated.

Noise less than 10 electrons possible.

Charge transfer is excellent.

\section{Thomson 384 X 576:}

23 micron pixels; $8.8 \times 13.2 \mathrm{~mm}$ format.

Unthinned, frontside-illumination; phosphor for UV.

10 electron noise and good transfer efficiency.

Versions buttable on three sides are now being developed, allowing arrays of $2 \mathrm{X} \mathrm{N}$ to 
be fashioned.

\section{EEV 384 X 576:}

22 micron pixels; frontside illuminated.

Recent work toward thinning has been undertaken, and a new series of larger format devices (approx. 1150 pixels square) is being developed.

\section{Tektronix 512 X 512:}

27 micron pixels; $13.8 \mathrm{~mm}$ square format.

Noise expected to be about 10 electrons.

\section{Thomson 512 X 512 and $1024 \times 1024$ :}

Currently under development for scientific applications. 19 micron pixels; formats of $9.7 \mathrm{~mm}$ and $19.5 \mathrm{~mm}$ square.

Thinned version eventually is under active development.

\section{Reticon 400 X 1200:}

27 micron pixels; $10.8 \times 32.4 \mathrm{~mm}$ format.

Initial unthinned versions in development production.

First samples exhibit good transfer and low noise.

Thinned version is planned as soon as development is completed.

\section{Tektronix 2048 X 2048:}

Available next year (?)

Thinned, backside-illuminated.

27 micron pixels; $55.3 \mathrm{~mm}$ square format!!!

Expensive - about $100 \mathrm{~K} \$ ! ! !$

Huge burden on data processing.

Formation of contractual efforts for custom devices, drawing on the services of imager manufacturers and silicon foundries. This last option for the procurement of just the right device for a specific class of instrumentation may ultimately be the most rewarding for the field. It does however require a level of cooperation between institutions at present, as the costs for a new start are at the moment beyond what most of us can budget.

\section{New-technology imagers:}

Looking toward the more distant future, things are of course not clear at all. Proposals for the next generation of CCD (or other solid-state imager) have not been extensively pursued because we have had such a devilishly hard time getting good devices from the present generation of manufacturing technology, or perhaps more correctly, from the past generation of device fabricators. With a shift in the offing, new ideas will now start to be investigated. The three listed below are certainly not comprehensive, but may serve as examples for the audience. 
1. New output structures capable of resolving single electron signals. Even some of the older, less well optimized output designs have shown themselves capable of approaching this ideal, and there is no fundamental reason why this goal cannot be routinely achieved. The CCD would thus become at least statistically equal to a true photon counter down at the single photoelectron level, allowing work in even the lowest signalto-noise regimes.

2. Devices designed to operate in electronic inversion of the electrode-side surface. This can reduce dark current a hundred fold or more, allowing much higher temperatures to be used, even for long integrations. This in turn can bring both increased quantum efficiency and increased charge transfer efficiency.

3. CCD structures designed for ease of manufacturing and increased yield of usable clevices, while still retaining all the essential characteristics of precision imagers. Such techniques may ultimately make possible devices of very large format but without the very large price tags of present technology. 